\title{
Alteration in periodontal pathogens and periodontal status in patients with nasogastric tube feeding- A clinical and microbiological study
}

\author{
Jaisika Rajpal $^{1 *}$, Aakash Arora ${ }^{2}$, Prerna Arora $^{3}$, Ruchika Prasad $^{4}$ \\ ${ }^{\mathbf{1 , 3}, \mathbf{4}}$ Senior Consultant, ${ }^{2}$ Reader, ${ }^{\mathbf{1}}$ Private Practitioner, Ghaziabad, Uttar Pradesh, ${ }^{\mathbf{2}}$ Dept. of Oral and Maxillofacial Surgery, ${ }^{\mathbf{2}}$ Shree Bankey \\ Bihari Dental College and Research Centre, Ghaziabad, Uttar Pradesh, ${ }^{3}$ Private Practitioner, Lucknow, Uttar Pradesh, ${ }^{4}$ Private Practitioner, \\ Delhi, India \\ *Corresponding Author: Jaisika Rajpal \\ Email: drjaisikarajpal@gmail.com
}

\begin{abstract}
Introduction: The oral indigenous microbiota exists in a state of balance with the host, but the long-term use of tube feeding alters the intraoral conditions in general and periodontal microbiota in particular. The aim of this study was to examine the impact of nasogastric tube feeding on the periodontal microbiota and to explore the periodontal status in patients with nasogastric tube feeding. Method-In this study, the plaque microbiota of subjects fed enterally was compared comprehensively with that of subjects fed orally to characterize the oral indigenous microbiota of tube-fed patients. Thirty patients with nasogastric tube feeding (test group) and thirty patients fed orally (control group) were recruited for the study. Periodontal health status was assessed by plaque index and gingival bleeding by using William's periodontal probe. Microbiological evaluation was done by quantitative cultures of dental plaque 7 days after nasogastric tube insertion. In controls, cultures of plaque and buccal mucosa were done after 7 days of hospitalization.

Results: A high prevalence of potentially pathogenic isolations was found in tuboenterally fed patients in Group I as compared to Group II along with increased bleeding sites and plaque index.

Conclusion: On the basis of the observed results of the study, we can concluded that the risk for periodontal disease appeared to be significantly elevated in patients with nasogastric tube feeding.
\end{abstract}

Keywords: Nasogastric tube feeding, Periodontopathogenic bacteria, Microbial cultures.

\section{Introduction}

Enteral tube feeding is widely used to maintain nutrition in patients with a functional gastrointestinal tract but inadequate oral intake. Enteral feeding is a key component in the management of critically ill patients to tolerate feeding into the gastrointestinal tract. The reported benefits of enteral nutrition over parenteral nutrition include preservation of intestinal mucosa, optimal nutrient utilization, safer administration, and reduced cost. ${ }^{1}$ Increasing numbers of critically ill patients and elderly patients with oropharyngeal dysphagia require tubal enteral feeding in order to prevent aspiration and to maintain adequate nutrition and hydration. ${ }^{2}$ The oral indigenous microbiota exists in a state of balance with the host, but the use of tube feeding alters the intraoral conditions. ${ }^{3}$ Critically ill patients are vulnerable to aspiration because of decreased level of consciousness, altered gastrointestinal motility, slower gastric emptying, and presence of artificial airways. ${ }^{4,5}$ Patients can aspirate oral secretions or refluxed stomach contents containing tube-fed formula, ${ }^{6}$ this can involve the aspiration of bacteria from the oropharynx into the lower respiratory tract and regurgitation of gastric contents into the oropharynx. The pathogens are subsequently shed into the salivary secretions, together with oral bacteria, hydrolytic enzymes and pro-inflammatory cytokines. The absence of food passage results in an absence of mechanical clearance within the mouth and reduces saliva secretion. The mucosal surfaces often dry out, and dried sputum adheres to the palate. These ecological changes should affect the bacterial population of the indigenous microbiota. Unexpected bacteria may thrive in the disused oral cavity and the failure of the host defense systems to eliminate pathogens from the mucosal surface results in their proliferation, which is followed by infection and tissue destruction..$^{7-9}$ The progression of the disease is related to the colonization of different microorganisms; including Aggregatibacter actinomycetemcomitans as well as members of the so-called "red complex": Porphyomonas gingivalis, Tannerella forsythia and Terponema denticola. ${ }^{10}$ The detection of bacterial species helps to predict disease prognoses and to provide treatment strategies. Thus the hypothesis of the present study was to assess total bacterial count of different periodontopathic organisms which may be present in plaque of patients on nasogastric tube feeding and to explore the periodontal status of such patients. In this study, the plaque microbiota of subjects fed enterally was compared comprehensively with that of subjects fed orally to characterize the oral indigenous microbiota of tube-fed patients.

\section{Aim and Objectives}

To date, only a few studies have been conducted to determine the periodontal status of enterally fed patients. Since, data concerning periodontal health status and specific oral care requirements of such patients is rare, this study was undertaken, with the following aims and objectives:

1. To clinically analyze the periodontal health status of hospitalized patients with tuboenteral feeding.

2. To assess total bacterial count of different periodontopathic in mature dental plaque formed after 7 days of naso gastric tube feeding (NGT).

3. Detection of "red complex" bacteria -Porphyomonas gingivalis, Tannerella forsythia and Terponema denticola to predict disease progression 
4. To provide guidelines for such patients regarding oral health care measures.

\section{Materials and Methods}

This study was carried out at Subharti Medical College and Hospital, after obtaining institutions ethical clearance. The patient population for this study was nonconsecutive, unselected patients admitted to the medical ICU during a 6 month period and controls were age matched patients from the same ICU admissions. This cross-sectional retrospective study included a study population of 60 patients. Thirty patients with nasogastric tube feeding (test group- Group 1) and thirty patients fed orally (control group-Group 2) were recruited for the study. Inclusion criteria- 1) patients admitted in ICU and put on parenteral feeding using nasogastric tube, 2) Patients aged 20 to 80 years old and had $\geq 20$ natural teeth. Exclusion criteria- 1) Patients with existing periodontal disease, 2) Patients with a history of any periodontal treatment in the past 6 months, 3) Smokers, 4) Patients with advanced cancer of any kind and those after irradiation to the head and neck.

Periodontal health status was assessed by measuring plaque index and gingival bleeding by using William's graduated probe. Microbiological evaluation was done by quantitative cultures of dental plaque after 7 days of placement of nasogastric tube. In controls, cultures of plaque were done after 7 days of hospitalization. Clinical examination was performed using a torch for illumination, a mouth mirror, straight explorer and a William's periodontal probe. Indices were recorded for four surfaces (M, B, D, and L) of the index teeth. No disclosing agent was used for the assessment of plaque levels and only clinically obvious plaque was recorded using the criteria listed by Silness and Loe in 1964 (score $0=$ no dental plaque in the gingiva area, score $1=$ a film of dental plaque adhering to the free gingival margin (only upon probing), score $2=$ moderate accumulation of dental plaque at the gingival margin (seen by naked eye), score $3=a b u n d a n t$ dental plaque in the gingival margin). ${ }^{11}$ Similarly, the gingival disease assessment was done using Gingival index and this parameter was estimated on a scale of 0-3 according to Loe and Silness in $1963 .^{12}$ Overall plaque score and gingival disease status were calculated for each patient by dividing the sum of the plaque scores by the total number of sites sampled.

\section{Sample collection}

For plaque sampling, selected teeth and adjacent area were isolated with cotton rolls, to prevent the contamination of the samples with saliva. The plaque samples were taken using one sterile paper point ISO \#40 which was inserted slowly with a sterile dental tweezer deep into the pocket until tissue resistance was felt. The paper point was left for 20 sec., (Fig. 1) and then it was carefully removed without touching the adjacent unrelated tissues and placed into a special sterile container and sent for microbiological examination. (Fig. 2)

\section{Culture method}

Samples were serially diluted using half strength thioglycolate medium. Appropriate dilutions $(0.05 \mathrm{ml})$ were then plated in triplicate onto trypticase soya agar \& nutrient agar. The agar plates were placed immediately in an anaerobic chamber and maintained at $37^{\circ} \mathrm{C}$ for up to 5 days, except for nutrient agar, which was incubated aerobically in a standard incubator for 3 days. Morphologically distinct bacterial colonies were counted, subcultured, and archived at $-80^{\circ} \mathrm{C}$ for subsequent identification. After an incubation period, the plates were observed for microbial growth. The total number of colony forming units (CFU) for each plate was assessed for each sample using a colony counter. (Fig. 3) The number of CFUs is related to the viable number of bacteria in the sample.

C.F.U $/ \mathrm{mL}$ original sample $=$ Number of colonies $/$ plate $\mathrm{x}$ $(1 / \mathrm{mL}$ aliquot plated) $\mathrm{x}$ dilution factor

The isolated bacterial species were morphologically characterized by gram staining. The red complex bacteria were tabulated as present or absent in the sample.

\section{Statistical analysis}

Statistical analysis was performed using the SPSS software. Descriptive presentation of the data was followed by comparative statistical processing using Student's t-test or chi-square test. Pearson's coefficient was used for correlation studies. $\mathrm{P}<0.05$ was considered significant.

\section{Results}

The demographic data of the study population is tabulated in Table 1. The analysis of the data signified that the average age of group 1(test group) is 48.83 and group 2 (control group) is 45.51 respectively. The male to female ratio in both the groups was 43:57. In Group 1 and 40:60 in Group 2

The plaque score range of group 1 is $1.2633 \pm 0.6390$ and control group is $0.904 \pm 0.5857$ and $\mathrm{P}$ value is $\mathrm{P}<0.05$ which signifies that there is a significant difference between mean values of plaque score of experimental group and control group (Fig.4). Experimental group has more average values than control group.

The bleeding score range of group 1 is $1.300 \pm 0.6676$ and group 2 is $0.983 \pm 0.5984$, and $\mathrm{P}<0.05$, which signifies that there is a significant difference between the mean value of bleeding score of experimental group and control group (Fig. 5). Experimental group has more average value than control group.

Chi. Square test was performed to test the association between bacterial isolations in both the groups which was found to be significant. Noteworthy is the high prevalence of pathogenic isolations in Groups 1 i.e. $80 \%$ and, as compared with $27 \%$ in the control group $(\mathrm{P}<.05)$. Porphyromonas gingivalis was highly prevalent, mainly in the NGT fed patients $(37 \%$; p, .001) as compare to $20 \%$ in orally fed group. Treponema denticola and Tanerella forsythia were also isolated, primarily from the NGT fed patients (Table 2). Not surprisingly, the NGT fed patients had a higher prevalence of each pathogenic gram negative bacteria as compared with those on oral feeding. No 
correlation was found between the length of stay in the ICU or the duration on tube feeding and bacterial isolations. The presence of other pathogen is also significant in both the groups although a detailed study is recommended for other pathogens.

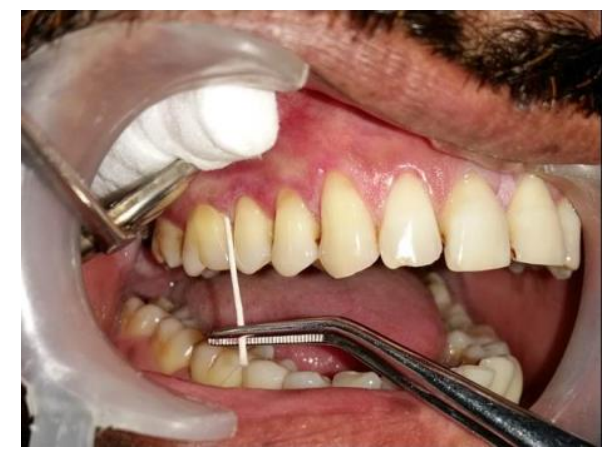

Fig. 1: Plaque sample collection with paper point

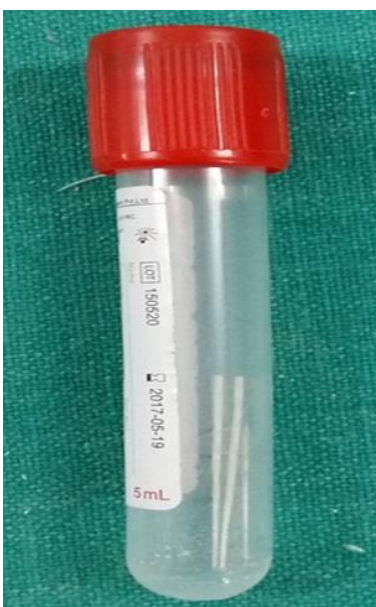

Fig. 2: Sample transferred in sterile container and sent for microbiological examination

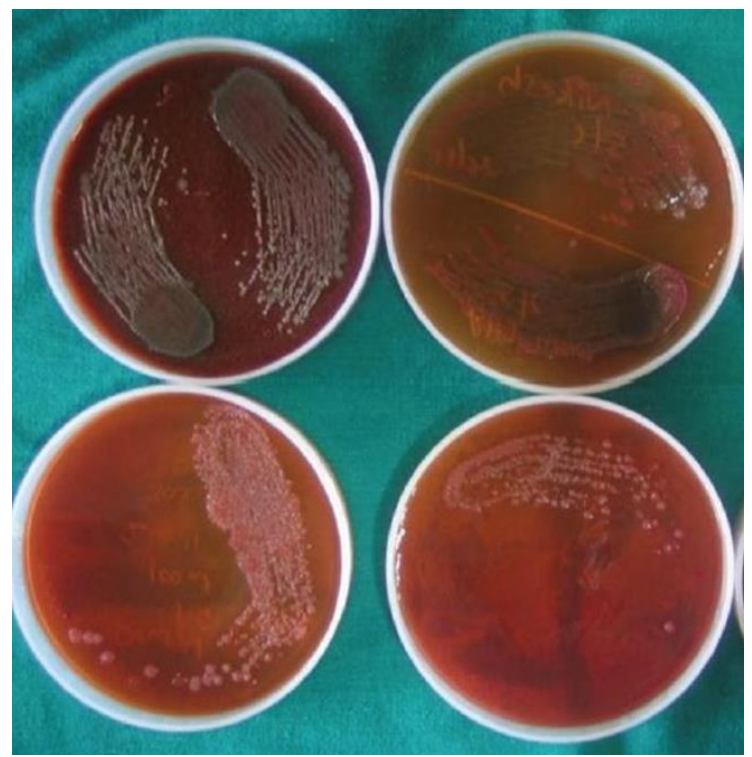

Fig. 3: Nutrient media with colony forming units

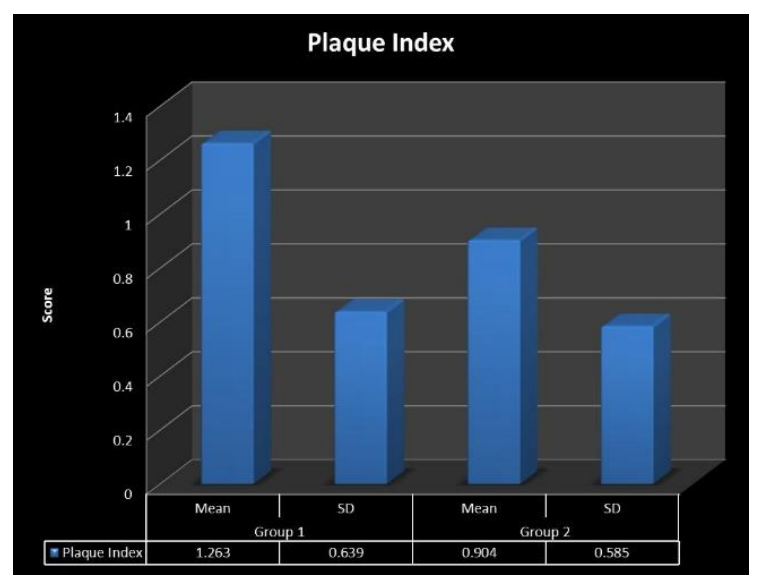

Fig. 4: Graph comparing plaque index of Group 1 and Group 2

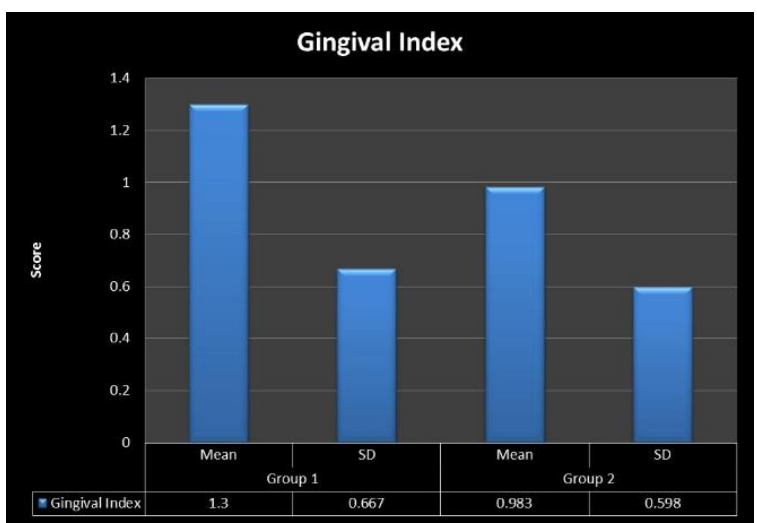

Fig. 5: Graph comparing plaque index of Group 1 and Group 2

Table 1: Demographic data

\begin{tabular}{|l|c|c|c|c|}
\hline & \multicolumn{2}{|c|}{ Group 1 } & \multicolumn{2}{c|}{ Group 2 } \\
\hline Gender & Male & Female & Male & Female \\
\hline & 17 & 13 & 18 & 12 \\
\hline Age & Min. age & Max. age & Min. age & Max. age \\
\hline & 19 yrs. & 80 yrs. & 19 yrs. & 80 yrs. \\
\hline
\end{tabular}

Table 2: Isolation rates of pathogenic flora in patients

\begin{tabular}{|l|c|c|c|c|c|}
\hline Isolation & Group 1 & & Group 2 & & P \\
\cline { 2 - 6 } & No. & $\%$ & No. & $\%$ & \\
\hline $\begin{array}{l}\text { Porphyromonas } \\
\text { gingivalis }\end{array}$ & 11 & $37 \%$ & 6 & $20 \%$ & $<.001$ \\
\hline $\begin{array}{l}\text { Tanerella } \\
\text { forsythia }\end{array}$ & 7 & $23 \%$ & 0 & $0 \%$ & $<.028$ \\
\hline $\begin{array}{l}\text { Treponema } \\
\text { denticola }\end{array}$ & 4 & $13 \%$ & 1 & $3 \%$ & $<.001$ \\
\hline Any pathogen & 24 & & 8 & $27 \%$ & $<.05$ \\
\hline
\end{tabular}

\section{Total bacterial viable count}

Quantitation of total bacteria was evaluated in order to determine the effect of nasogastric tube on bacterial colonization. The occurrence of bacteria was high in Group 1 as compared to that found in Group 2. In first group mean of total bacterial count was $7.5 \times 10^{12} \mathrm{CFU} / \mathrm{ml}$ and that in Group 2 was $5.6 \times 10^{10} \mathrm{CFU} / \mathrm{ml}$. 


\section{Discussion}

Results of our study state that hospitalized patients with nasogastric tube have consistently poor periodontal status compared to controls. Cases with NGT had significant difference in PI, GI and red complex bacteria compared to controls $(p<0.05)$. Plaque and gingival bleeding scores on NGT patients were significantly high as compared to the orally fed patients; these results were consistent with the previous studies done by Thoden van et al $1984^{13}$ and TE Lloyd et al 2011. ${ }^{14}$

The oral microbiota in the subgroup of prolonged nasogastric tube-fed elderly patients has scarcely been investigated. Early studies reported on the tendency of gram- negative bacteria to colonize the oropharynx of hospitalized patients ${ }^{15,16}$ but mentioned that nasogastric tube-fed patients were not included because of the small numbers at that time. ${ }^{15}$ Another study mentioned the presence of gram-negative bacteria in surgical patients with nasogastric feeding for a short time (48 \pm 72 hours). ${ }^{17}$ However, these were not comparative studies. To our knowledge, the present study is the first to make a comparative examination of NGT and orally fed elderly patients with respect to periodontal pathogens.

Reliable clinical indices to determine periodontal disease status should be associated with specific periodontal pathogens such as microorganisms of the red complex: Porphyromonas gingivalis, Treponema denticola, and Tannerella forsythia. ${ }^{18}$ These species are associated with periodontal tissue breakdown and active lesions of destructive periodontal disease. ${ }^{19}$ The oral flora of critically ill patients with nasogastric feeding differs from that of healthy individuals and contains organisms that can rapidly cause periodontal disease. Within 48 hours of tube insertion, the composition of the oropharyngeal flora of patients undergoes a change from the usual predominance of grampositive pathogens to predominantly gram-negative organisms, constituting more virulent flora, including pathogens that cause periodontitis. ${ }^{20}$ Also, increased levels of proteases in the oral secretions of critically ill patients removes from their epithelial cell surfaces a glycoprotein substance called fibronectin. Normally, fibronectin is present on cell surfaces and acts as a host defense mechanism, blocking pathogenic bacterial attachment to oral and tracheal mucus membranes. This depletion of fibronectin allows cell receptor sites to replace normal flora with virulent pathogens such as Porphyromonas gingivalis on the tooth surface. ${ }^{21}$

If the intubated patient does not receive effective, comprehensive oral hygiene, dental plaque and hardened bacterial deposits develop on the teeth within 72 hours. This is followed by emerging gingivitis, gum inflammation, infection and a subsequent shift from primarily Streptococcus and Actinomyces spp. to increasing numbers of aerobic gram negative bacilli. ${ }^{22}$ Since adhesion to a surface in the mouth is important for the continued existence and proliferation of organisms, bacteria which attach to the tooth surface gradually coalesce to produce a biofilm and after further development, lead to the formation of dental plaque. ${ }^{17}$

The main finding in our study is the significantly higher rate of pathogenic isolations from the plaque of tuboenterally fed patients. GNB have been isolated from $80 \%$ of the NGT fed patients as opposed to only $27 \%$ of the orally fed group (p, <0.05). The prevalence of Porphyromonas gingivalis in the plaque was extremely high in those patients fed by NGT. Our results are consistent with the view that it is the mechanical clearance associated with proper chewing and swallowing that provides the main defense against oral pathogenic contamination.

The higher incidence of gram negative bacteria (GNB) colonization in the NGT fed patients as compared with those fed orally may have several explanations. The fact that these bacteria were only rarely isolated from the orally fed patients in our study suggests that natural oral feeding itself may be a factor in preventing their colonization. In the absence of mastication and food passage, coupled with altered saliva composition, the oropharynx of nasogastric feeding patients could be devoid of its natural protective mechanism. The long term absence of food passage is an extremely abnormal situation for the oral indigenous microbiota. While fluid and carbohydrate supply is stopped, mechanical clearance by mastication drastically decreases. A recent publication emphasized the importance of these elements to the oral clearance for the preventing of pathogenic oropharyngeal colonization in the elderly. ${ }^{23}$ The presence of an abiotic surface such as the tube itself may constitute a site of biofilm formation, bolstering the growth of periodontal bacteria. Alterations of normal salivary flow and composition may also be related to the colonization of the oropharynx by gram-negative bacteria. ${ }^{24}$ Studies by Dennesen et al. have documented a reduced salivary flow in intubated patients with resultant mucositis resulting in the increase of bacterial load in the oral secretions, allowing increased buildup of biofilm. ${ }^{25}$ Leibovitz et al in their study in 2003 reported the lower concentration of uric acid in the saliva of patients with nasogastric feeding and the resultant reduced antioxidant activity which could also contribute to the risk of colonization by periodontal pathogens. ${ }^{26}$ Moreover, an association has been reported between the short-term use of NGTs and sinusitis. ${ }^{27}$ Prolonged use could have a higher impact on the pathological colonization of the sinuses, leading to chronic sinusitis that may be a source of pathogenic bacteria. In addition, the disturbed balance of beneficial and detrimental bacteria in the indigenous microbiota, or dysbiosis, has recently attracted attention in regard to the development of mucosal inflammation. ${ }^{28}$ Furthermore, these patients are also immuno-compromised because of disease or medication and thus vulnerable to colonization by multidrug-resistant microorganisms. ${ }^{29}$

Our study clearly showed the significantly higher rate of potentially pathogenic periodontal bacteria cultured from the oral cavity of patients on long-term nasogastric feeding as compared to oral feeding. 


\section{Conclusion}

Taken together, these findings indicate that prolonged nasogastric feeding is associated with alterations in the flora of the oral cavity. One should pay careful attention to the bacterial populations in the oral cavity with the use of feeding tubes. Lack of attention to oral hygiene results in increase in mass and complexity of dental plaque, which will lead to interaction between indigenous plaque bacteria and respiratory pathogens. These changes could be related to the risk of aspiration and aspiration pneumonia in longterm care elderly patients fed by nasogastric tube. While the benefits of oral care in preventing pneumonia in elderly adults are well documented, oral care is generally neglected in patients receiving tube feeding due to the erroneous impression that their oral cavities are not used. Rather, our results suggest that tube fed patients need aggressive oral care to prevent the overgrowth of a disturbed microbiota, even if such care might be ineffective at restoring the normal microbiota. The current protocol within the intensive care unit is for cleaning the oral cavity twice daily cleaning with a toothbrush and toothpaste. However, the actual methods used for mouth cleaning by intensive care staff varied more widely, with some staff using swabs dipped in chlorhexidine mouthwash and on occasions, oral care was forgotten. Many nurses are also discouraged from cleaning the mouth because the endotracheal tube makes performing this task difficult and there is a fear of dislodging the tube and precipitating cardiac arrest. Even when procedures are in place, mouth care is often performed poorly or inconsistently. Oral care is needed for hospitalized patients with NGT as they are at greater risk for periodontal infections and subsequent VAP. The ICU patients are not able to perform oral care for themselves so nursing staff needs to be responsible for providing oral care. In order for nurses to feel comfortable providing oral care, training should be increased and include evidence-based rationale for assessment and care. Lastly, an oral assessment tool should be implemented when providing ICU care for patients. It is a reliable guide for nurses to assess the oral care needed for patients and an easy guide for an oral examination. When nurses are educated on providing oral care using an oral assessment tool, they can better care for their patients as a whole and reduce the risk of other illnesses and conditions from occurring.

\section{Limitations}

1. The major limitations of our study were the pre-post test design and the differences between the treatment and control groups, including diagnosis, severity of illness, and other factors.

2. This study was cross-sectional and thus cannot unequivocally demonstrate that feeding tube placement results in oral dysbiosis. A follow-up study would clarify the environmental trigger of the dysbiosis associated with tube feeding and may lead to the development of a novel approach to prevent the oral dysbiosis in tube feeding.

3. A smaller than anticipated sample size.
4. Many of the patients had co-morbidities such as depression and dementia which could also have a major influence on oral hygiene and gingival/periodontal condition.

\section{Conflict of interest}

None.

\section{Source of funding}

None.

\section{References}

1. EL-Baz N. The effect of two methods of intermittent enteral feeding on critically ill patients. M.Sc. Thesis. University of Alexandria, Faculty of Nursing, Egypt; 2002.

2. Guenter P, Jones S, Swed RM. Delivery systems and administration of enteral nutrition. In: Rombeau I, Rolandelli R, eds. Enteral and Tube Feeding. 3rd edn. Philadelphia: WB Saunders Co., 1997:240-53.

3. Yamaya M, Yanai M, Ohmi T, Arai H, Sasaki H. Interventions to prevent pneumonia among older adults. J Am Geriatr Soc 2001;49:85-90.

4. Daniels R., Grendell R. and Wilkins F. (2010). Nursing fundamentals: Caring \&clinical decision making. $2^{\text {nd }} \mathrm{Ed}$., USA: Saunders Elsevier Co.; 1167-71.

5. Sanko JS. Aspiration assessment and prevention in critically ill enterally fed patients: Evidence-based recommendations for practice. Gastroenterol Nurs 2004;27(6)279-85.

6. Serna ED, Mccarthy MS. Heads up to prevent aspiration during enteral feeding. Nurs 2006;36(1):76-7.

7. Estes RJ, Meduri GU. The pathogenesis of ventilator associated pneumonia: I. Mechanisms of bacterial trans colonization and airway inoculation. Intensive Care Med 1995;21:365-83.

8. Scannapieco FA, Mylotte JM. Relationships between periodontal disease and bacterial pneumonia. J Periodontol 1996;67(Suppl. 10);1114-22.

9. Fagon J-Y, Chastre J. Severe exacerbations of COPD patients: The role of pulmonary infections. Semin Respir Infect 1996;11:109-18.

10. Harper-Owen R, Dymock D, Booth V, Weightman AJ, Wade WG. Detection of unculturable bacteria in periodontal health and disease by PCR. J Clin Microbiol 1999;37:1469-73.

11. Silness J, Loe H. Periodontal disease in pregnancy. II. Correlation between oral hygiene and periodontal condition. Acta Odontol Scand 1964;22:112-35

12. Loe H, Silness J. Periodontal disease in pregnancy I. Prevalence and severity. Acta Odontol Scand 1963;21:533-51.

13. Thoden Van Velzen SK, Abraham-Inpijn L, Moorer WR. Plaque and systemic disease: a reappraisal of the focal infection concept. J Clin Periodontol 1984;11:209-20.

14. Lloyd TE, Frost PJ, Rees JS. A pilot audit of oral health in mechanically ventilated critically ill patients. J Disabil Oral Health 2011;12/3 114-20.

15. Pajukoski H, Meurman JH, Odont D, Snellman-Grohn S, Sulkava R. Oral heath in hospitalized and non-hospitalized community dwelling elderly patients. Oral Surg Oral Med Oral Pathol 1999;88:437-43.

16. Valenti WM, Trudell RG, Bentley DW. Factors predisposing to oropharyngeal colonization with gram-negative bacilli in the aged. N Engl J Med 1978;298:1108-11.

17. Irwin RS, Whitaker S, Pratter MR, Millard CE, Tarpey JT, Corwin RW. The transiency of oropharyngeal colonization with gram-negative bacilli in residents of a skilled nursing facility. Chest 1982; 81:31-5. 
18. Socransky SS, Haffajee AD, Cugini MA, Smith C, Kent RL. Microbial complexes in subgingival plaque. J Clin Periodontol 1998;25(2):134-44.

19. Kuboniwa M, Amano A, Kimura KR, Sekine S, Kato S, Yamamoto Y, et al. Quantitative detection of periodontal pathogens using real-time PCR with TaqMan probes. Oral Microbiol Immunol 2004;19(3):168-76.

20. Berry AM, Davidson PM. Beyond comfort: Oral hygiene as a critical nursing activity in the intensive care unit. Intensive Crit Care Nurs 2006;22(6):318-28.

21. Munro CL, Grap MJ. Oral health and care in the intensive care unit: state of the science. Am J Crit Care 2004;13(1):25-33.

22. Bagg J ea. The oral microflora and dental plaque. Essentials of microbiology for dental students. Oxford: Oxford University Press; 1999;229-310.

23. Preston AJ, Gosney MA, Noon S, Martin MV. Oral flora of elderly patients following acute medical admission. Gerontol 1999;45:49-52.

24. Palmer LB, Albulak K, Fields S, Filkin AM, Simon S, Smaldone GC. Oral clearance and pathogenic oropharyngeal colonization in the elderly. Am J Respir Crit Care Med 2001;164:464-8.

25. Dennesen P, van d, V, Vlasveld M, Lokker L, Ramsay G, Kessels A, et al. Inadequate salivary flow and poor oral mucosal status in intubated intensive care unit patients. Crit Care Med 2003;31(3):781-6.

26. Leibovitz A, Plotnikov G, Habot B, Rosenberg M, Wolf A, Nagler R, et al. Saliva Secretion and Oral Flora in Prolonged Nasogastric Tube-Fed Elderly Patients. Isr Med Assoc J 2003;5:329-32.

27. George DL, Falk FS, Umberto Meduri G. Nosocomial sinusitis in patients in the medical intensive care unit: a prospective epidemiological study. Clin Infect Dis 1998;27:463-70.

28. Sartor RB. 2008. Therapeutic correction of bacterial dysbiosis discovered by molecular techniques. Proc Natl Acad Sci 2008;105:16413-4.

29. Amaral SM, Côrtes AQ, Pires FR. Nosocomial pneumonia: importance of the oral environment. J Bras Pneumol 2009;35(11):1116-24

How to cite: Rajpal J, Arora A, Arora P, Prasad R. Alteration in periodontal pathogens and periodontal status in patients with nasogastric tube feeding- A clinical and microbiological study. IP Int J Periodontol Implantol 2020;5(1):19-24. 